\section{ESTUDIO PALINOLÓGICO DE LOS SEDIMENTOS ARQUEOLÓGICOS DEL YACIMIENTO DEL LLANETE DE LOS MOROS (CÓRDOBA)}

\author{
PALYNOLOGICAL STUDY OF \\ ARCHAEOLOGICAL SEDIMENTS FROM \\ LLANETE DE LOS MOROS (CORDOBA)
}

\section{PILAR LÓPEZ GARCÍA (*) \\ JOSÉ ANTONIO LÓPEZ-SÁEZ (**)}

\section{RESUMEN}

Se presentan los resultados del análisis paleopalinológico llevado a cabo en el yacimiento arqueológico del Llanete de los Moros, con una cronología arqueológica, encuadrable en la Edad del Bronce, y que lo sitúa climáticamente en el Subboreal-Subatlántico. Se pone de manifiesto una amplia representación de especies nitrófilas así como una cobertura arbórea poco manifiesta. Se comenta la presencia de Vitis y Olea.

\begin{abstract}
This paper presents a pollen analysis from excavations at the Bronze Age site of Llanete de los Moros, with special reference to Vitis and Olea, and the presence of anthropic plants, enabling the definition of distinct periods of the Holocene. The action of man seems to be present throughout the diagram.
\end{abstract}

(*) Departamento de Prehistoria. C.E.H. C.S.I.C. Duque de Medinaceli 6. 28014 Madrid.

(**) Departamento de Biología Vegetal I. Facultad de Biología. Ciudad Universitaria $s / n$. Universidad Complutense. 28040 Madrid.

El artículo fue remitido en su versión final el 7-X-94.
Palabras clave: Palinología. Subboreal. Subatlántico. Holoceno. Arqueología. Córdoba.

Key words: Palynology. Subboreal. Subatlantic. Holocene. Archaeology. Cordoba.

\section{INTRODUCCIÓN}

La escasez de análisis polínicos para el Holoceno ibérico, mayor en el caso de yacimientos arqueológicos (Asquerino, 1987) queda igualmente de manifiesto en la recopilación de López García (1978). Es por ello, que resulta no sólo difícil sino imposible establecer correlaciones y delimitar características ambientales de un momento dado de la Prehistoria andaluza, más aún si cabe, dada la gran variedad de ecosistemas y por ende, de formaciones vegetales de Andalucía, lo que implicaría un número mucho mayor de este tipo de trabajos para conseguir al menos conclusiones relativamente válidas acerca del paisaje andaluz a lo largo del Cuaternario.

Los yacimientos arqueológicos plantean más problemas a la hora de la interpretación de los análisis polínicos que los depósitos de turba o 
detríticos, debido a la propia naturaleza del sedimento y del depósito. Inconvenientes que presentan son la filtración del polen con contaminación de niveles más viejos, destrucción diferencial del mismo, hiatus sedimentarios frecuentes e importantes y gran pobreza polínica (Couteaux, 1977; Dupré, 1979).

Aunque la sedimentación polínica en grutas no es muy conocida y hay que cuidar la interpretación de sus espectros polínicos por la posibilidad de distorsión, mediante acciones humanas o animales de aporte de coprolitos, desechos, etc, muchos de estos inconvenientes también existen en turberas y lagos pero más atenuados (Vázquez, 1992). En todo caso, la interpretación de los espectros fósiles de yacimientos arqueológicos, aunque complicada, proporciona una información valiosa. La escasez de turberas y otros medios lacustres, sobre todo en la región andaluza, donde exceptuando la provincia de Huelva, el resto son realmente pobres en este tipo de medios, se solventa gracias a la posibilidad complementaria de poseer análisis de sedimentos arqueológicos, que a su vez pueden verse ayudados por otras investigaciones sedimentológicas, antracológicas, paleontológicas, etc. En la provincia de Córdoba únicamente disponemos de dos análisis esporopolínicos, uno en una turbera de Sierra Madrona (Díaz Fernández, 1992) y otro en sedimentos arqueológicos del yacimiento de Ategua (López García, 1986).

En este trabajo presentamos los resultados del análisis palinológico llevado a cabo en el yacimiento del Llanete de los Moros, con el que pretendemos contribuir a un mejor conocimiento de la vegetación durante la Prehistoria de Andalucía.

\section{LOCALIZACIÓN: EL MEDIO FÍSICO Y LA VEGETACIÓN}

El yacimiento del Llanete de los Moros se encuentra situado en la localidad cordobesa de Montoro, en una posición estratégica desde el punto de vista fisiográfico, siendo sus coordenadas las siguientes: $0^{\circ} 41^{\prime}$ de longitud W. y $38^{\circ} 1^{\prime}$ de latitud $\mathrm{N}$.

En coordenadas Lambert: $x$ (539.131) y (381.345), según la hoja 903 del Mapa de la Edición militar a escala 1:50000 (Martín de la Cruz, 1978: 105) (Fig. 1).

\section{T. P., 51, n. $^{\circ} 2,1994$}

(c) Consejo Superior de Investigaciones Científicas Licencia Creative Commons 3.0 España (by-nc)
Se halla limitado al Norte, Este y Oeste por uno de los meandros del río Guadalquivir, asentándose sobre un cerro de $246 \mathrm{~m}$. de altura, a caballo entre la vertiente sur de Sierra Morena y la Campiña.

Esta posición le confiere un importante privilegio desde el punto de vista natural y paisajístico. Dentro del entorno físico del yacimiento se distinguen cuatro unidades paisajísticas: la sierra. el piedemonte, la campiña y la vega. Cada una de ellas, con características litológicas y climáticas propias, son soporte de una vegetación potencial diferente.

El clima de la zona puede definirse como templado-cálido, en base a veranos muy secos, caracterizados por las altas temperaturas y la falta de precipitaciones, así como por inviernos suaves, posibilitando por ello la entrada de especies termófilas en la vegetación del entorno. La precipitación media anual se acerca a los 550 $\mathrm{mm}$ y la amplitud térmica es de $18^{\circ} \mathrm{C}$. Por tanto, bioclimáticamente, la zona de estudio se

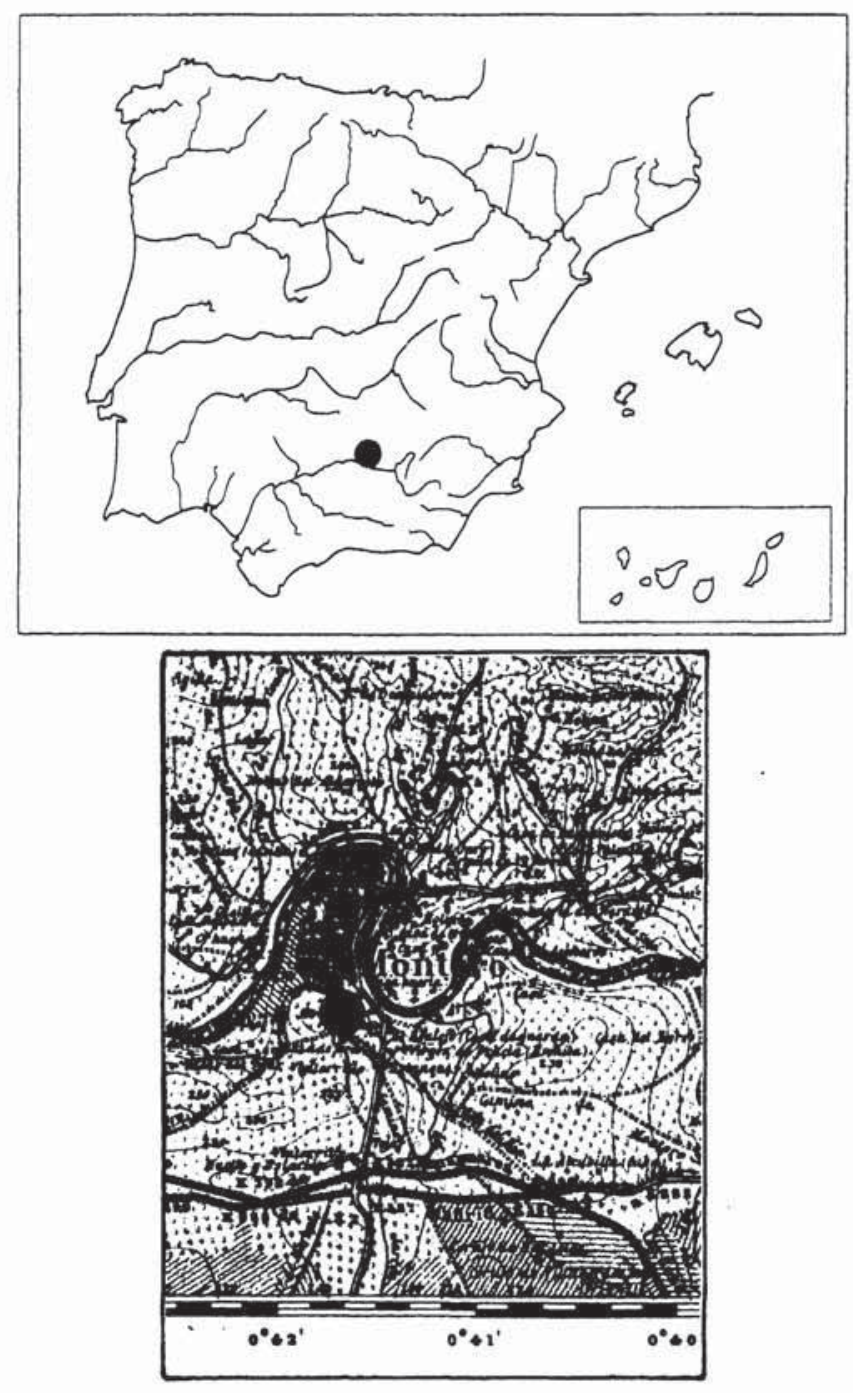

Fig. 1. Localización del yacimićnło. 
encuadra dentro del piso mesomediterráneo cálido, aunque en zonas fluviales, donde la termicidad es mayor, aparecen formaciones termomediterráneas de ombroclima seco-subhúmedo (Rivas Martínez, 1987).

Desde el punto de vista edafológico, existe una gran variedad de suelos, en función del tipo de sustrato, principalmente ácido, y con distinto grado de evolución. Se desarrollan rankers en la sierra, suelos pardos alóctonos sobre el piedemonte, dedicados tradicionalmente a la dehesa de encina y alcornoque $y$, actualmente ocupados por el olivar y coníferas de repoblación (MAPA, 1977). En las terrazas del río se desarrollan suelos rojos calizos y suelos diluviales sobre sus márgenes. En la campiña los suelos son margosos de tipo rendziforme.

En consonancia con los suelos y el clima, la vegetación climácica se corresponde con los carrascales termomediterráneos silicícolas de la asociación mariánico monchiquense Myrto communis-Quercetum rotundifoliae Rivas Goday 1959 em., y con la asociación bética algarviense basófila de Smilaci mauritanicae-Quercetum rotundifoliae Barbero, Quézel \& Rivas Martínez, sobre suelos rojos calizos. Ambas son de ombroclima seco-subhúmedo y se desarrollan en ambas vegas del Guadalquivir. De manera general, se manifiestan en su faciación termófila con Pistacia lentiscus L. y Myrtus communis L. (Peinado Lorca y Rivas Martínez, 1987).

Por encima de estas formaciones termomediterráneas, ceñidas a las zonas más bajas de la vega, de influencia fluvial, se encuentra el encinar con piruétanos, mesomediterráneo silicícola luso-extremadurense o Pyro bourgaeanaeQuercetum rotundifoliae Rivas Martínez 1987, que se extiende por todo el piedemonte serrano. La degradación de dicho encinar, predominantemente por la introducción del olivo, permite la entrada en el encinar de especies propias de sus etapas seriales degradativas. Es por ello frecuente, que junto a la encina, se encuentren Cistus ladanifer L., Quercus coccifera L., Phillyrea angustifolia L., Cytisus multiflorus (L'Hér.) Sweet, Retama sphaerocarpa L., Genista hirsuta Vahl., etc. En la actualidad, el dominio climácico de tal encinar está siendo utilizado para labores forestales y explotación del olivar.

En la campiña, se desarrolla la asociación basófila del encinar mariánico monchiquense
Paeonio coriaceae-Quercetum rotundifoliae $\mathrm{Ri}$ vas Martínez 1984. con Pistacia lentiscus en su faciación termófila. Actualmente se encuentra sustituído por cultivos de olivos, vid y cerealísticos, así como por algodón y girasol. Al localizarse en un territorio tan amplio, con gradientes ecológicos muy variables, la diversificación de esta serie es muy grande, dando lugar a numerosas variaciones (Gómez Mercado y Valle Tendero, 1988). Es típica la estratificación de estos bosques cuando se desarrollan sobre suelos profundos, distinguiéndose cuatro estratos: el formado básicamente por encinas, que al unir sus copas crean un microclima nemoral, mucho más fresco que el macroclima general reinante. Un segundo estrato agruparía plantas trepadoras y lianas tales como Asparagus acutifolius L., Lonicera etrusca G. Santi, Clematis flammula L., Hedera helix L., etc. El tercer estrato arbustivo se vería formado por un denso matorral de $G e$ nista cinerea (Will.) DC. subsp. speciosa R. Goday \& T. Losa ex R. Martínez et alii y Rhamnus alaternus L. Finalmente, el cuarto estrato lo compondrían un dosel de herbáceas y Paeonia coriacea Boiss., Bupleurum rigidum L., así como una alfombra de musgos que nos acerca a esa idea de las húmedas condiciones microclimáticas del interior de tales formaciones. No obstante, es casi imposible encontrar tal tipo de bosques en la actualidad, reducidos en su totalidad a pequeños restos de esas antiguas formaciones forestales. En zonas de alta explotación ganadera, el bosque ha sido conducido a un adehesamiento extremo, implantándose comunidades retamoides en la que domina la retama y la genista cinérea. La situación más común es encontrar etapas degradativas del tipo chaparralcoscojar, e incluso tomillares de marcado carácter nitrófilo donde se hacen dominantes Santolina rosmarinifolia L., Santolina chamaecyparissus L. y Thymus zygis L.

Todas las formaciones descritas han seguido en general una dinámica regresiva, de tal modo que hoy en día sólo persisten vestigios a modo de "bosques isla", regulados por la intervención humana, sobre todo para explotaciones agropecuarias y silvopastoriles.

En zonas riparias, la vegetación típica es la olmeda de Aro italici-Ulmetum minoris R. Goday en G. López 1976, aunque su área potencial ha sido ocupada por cultivos y únicamente aparecen especies termófilas como Nerium olean$\operatorname{der}$ L. Los adelfares son formaciones domina- 
das fisionómicamente por la adelfa que, pueden llegar a constituir formaciones relativamente densas, sobre todo en ramblas pedregosas. Presentan su óptimo en la provincia corológica murciano-almeriense, aunque también existen en ciertos enclaves termófilos de la provincia luso-extremadurense (Sánchez Mata y de la Fuente, 1986), tales como la encajonada vega del Guadalquivir a su paso por Montoro.

\section{DATOS ARQUEOLÓGICOS}

El yacimiento, situado en la localidad de Montoro, en la provincia de Córdoba se compone de dos cerros, el denominado El Palomarejo y el Llanete de los Moros, ubicados en uno de los grandes meandros que describe el río Guadalquivir. Excavado el segundo de ellos, muestra un primer amesetamiento de 233 metros de altitud, seguido de otro a 10 metros por debajo del anterior, y un tercero, sin signos de ocupación, a 15 metros por debajo del segundo. Las excavaciones se efectuaron en la primera de las terrazas en los años 1980 y 1981. En la zona NE se realizaron tres cortes, R-1, R-2 y R-3. De este último proceden las muestras de este análisis.

Culturalmente se ha podido documentar una secuencia de hábitat prácticamente ininterrumpida, entre un momento tardío de la Edad del Cobre y los siglos II-III d.C. (Martín de la Cruz, 1978-9, 1984-5, 1987).

El corte R3 documenta una superposición directa entre los estratos previos y los que contienen los primeros elementos de Cogotas I. El estrato inicial se encuadra en una fase avanzada del Calcolítico. Hasta el denominado estrato V, se localizan materiales, correspondientes a la Edad del Bronce, produciéndose en este fase de la estratigrafía un gran derrumbe que sella y diferencia esta fase de la posterior, de cronología mucho mas moderna, en torno al 1100 a.C. (Martín de la Cruz, 1984-5).

\section{MATERIAL Y MÉTODOS}

Las muestras objeto de estudio proceden, como ya se ha indicado, del corte R-3, localizado en la zona NE del yacimiento. Sus dimensiones son de $8 \times 4 \mathrm{~m}$, alcanzando una profundi- dad relativa de unos $7 \mathrm{~m}$, respecto de la cota 0 $\mathrm{m}$. que dista de la superficie unos $0.30 \mathrm{~m}$.

Se tomaron un total de 42 muestras, cada 10 $\mathrm{cm}$, siguiendo una columna vertical, sólo interrumpida en los niveles superiores del corte, en el que, por aparecer restos de construcción de piedra, se tuvo que desplazar. Una vez superados, se retomó la columna vertical. De esta manera, se alcanzaron los $498 \mathrm{~cm}$ de profundidad. Los niveles superiores, hasta $98 \mathrm{~cm}$, no son considerados en el análisis, en base a la construcción, antes mencionada. Así mismo, se denota la presencia de tres niveles estériles situados entre los 498 y $458 \mathrm{~cm}, 418$ a $358 \mathrm{~cm}$ y finalmente, entre los 138 y los $98 \mathrm{~cm}$ de profundidad. De las 42 muestras señaladas, y dado que los resultados eran muy similares entre unas y otras, se optó por analizar la mitad, es decir 21 muestras, de las que figuran en el diagrama 10 , por ser las únicas que presentaban un número suficiente de táxones para poder ser consideradas válidas.

El método químico utilizado ha sido el clásico: tratamiento de las muestras con ácidos clorhídrico y fluorhídrico, seguido de álcalis (López García, 1984), con concentración del polen mediante flotación en licor denso de Thoulet (Goeury y Beaulieu, 1979), tinción de la muestra con fuschina básica y montaje en glicerol para su observación al microscopio óptico.

En la preparación de las muestras se utilizó así mismo un Agitador de Ultrasonidos (Branson, model 250/450 Sonifier), así como fibras de vidrio (type ALE) de $25 \mathrm{~mm}$ de Gelman Sciences.

La determinación de los tipos polínicos se realizó según Moore y Webb (1978 y 1992) y con la ayuda de la palinoteca del Laboratorio de Palinología del Centro de Estudios Históricos del C.S.I.C. (Madrid).

Con los datos obtenidos se elaboró el correspondiente diagrama polínico (Fig. 2), en el que figuran el número de palinomorfos por nivel arqueológico, los porcentajes de cada táxon así como la curva que relaciona el polen arbóreo (A.P.) y no arbóreo (N.A.P.). La confección del diagrama se realizó gracias al programa informático Mac. Draw II de Macintosh.

Tanto la preparación química, como la realización del diagrama ha corrido a cargo de Dña Rosario Macías, Ayudante de investigación del Laboratorio. 


\section{RESULTADOS Y DISCUSIÓN}

Una primera visión del diagrama polínico pone de manifiesto la inexistencia de un bosque como formación forestal densa, dado que éste siguió una evolución fuertemente regresiva.

Para facilitar nuestro estudio hemos subdivido los táxones identificados en el diagrama en tres grupos:

De una parte están aquellas especies relícticas, atribuíbles al encinar, bosque potencial existente previo a la deforestación, cuya presencia viene definida por Quercus rotundifolia. En todo momento los porcentajes de polen de Quercus no sobrepasan el $10 \%$ del A.P., aunque su presencia es relativamente constante a lo largo de todo el perfil, exceptuando claro está los niveles estériles.

Igualmente, se denota la presencia en el diagrama de táxones propios del cortejo florístico del encinar o de sus etapas seriales regresivas, caso de las Ericaceae, Cistaceae, Buxus y Lamiaceae a partir de los $318 \mathrm{~cm}$.

La presencia de Ephedra en los niveles más inferiores $(418 \mathrm{~cm})$ denota condiciones climáticas de marcada sequedad y termicidad. La identificación de algunos pólenes adscribibles a $\mathrm{Pi}$ nus halepensis en Carihuela (Granada) por Carrión (1991), confirman la mediterraneidad de este estatus vegetal, el cual junto a Ephedra fragilis debió ocupar los ambientes más xéricos.

Curiosamente, la presencia en el diagrama de pólenes de Vitis coinciden con un aumento porcentual de los niveles de Cistaceae, lo que es claramente indicativo de la degradación del encinar en favor de los cultivos vitivinícolas, apareciendo por ello etapas seriales tales como los jarales.

Aunque la presencia de Vitis es escasa porcentualmente, sí debemos destacar su constancia y coincidencia con una mayor degradación del medio forestal y por tanto, hacer unos comentarios al respecto.

Sin lugar a dudas, la presencia de polen de vid en análisis polínicos andaluces ha sido una de las mayores problemáticas con las que se ha enfrentado el conocimiento de la historia de la vegetación del sur de España. Stevenson (1985) y Stevenson y Moore (1988) citan la presencia de pólen de vid en un sondeo de la Laguna de las Madres (Huelva) fechado alrededor del 4480 BP, y en El Acebrón (Huelva) alrededor del 4340 BP respectivamente, testimonio inequí-

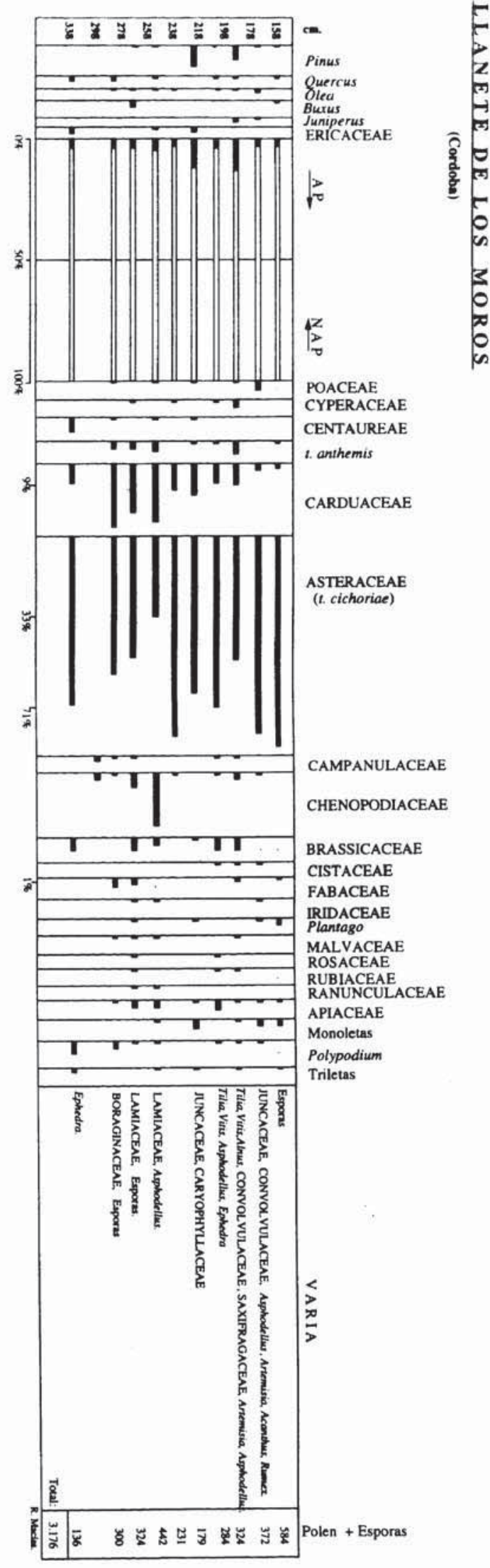

Fig. 2. Diagrama. 
voco del cultivo de vid en las inmediaciones, ya que sus altos porcentajes, superiores generalmente al $0.5-1.5 \%$ que supondría su presencia natural así lo atestiguan. En el registro de la historia vegetal de La Laguna de las Madres (Stevenson, 1985) hacia el $4480 \mathrm{BP}$, existe un periodo de viticultura bastante conspicuo. El autor llama la atención sobre la edad tan temprana en que se desarrolla el cultivo de Vitis. que contrasta con la opinión de otros autores que consideran que la vid fue introducida por los fenicios en la Península Ibérica hacia el $30(\%)$ $\mathrm{BP}$ o incluso por los romanos hacia el $2200 \mathrm{BP}$. El diagrama de la misma zona de MenéndezAmor \& Florschutz (1964) es semejante al de Stevenson (1985), siendo en torno al 4550 BP cuando aparecen aportes continuos de polen de Vitis. López García (1986) denota la presencia de Vitis en Almizaraque (Almería) durante el Subboreal, junto a cultivos cerealísticos y de regadío. La presencia asociada de polen de Vitis junto a indicadores del uso del fuego (Cistus) es muestra de su cultivo local (Stevenson y Moore, 1988). En este sentido, las afirmaciones de tal autor concuerdan con nuestras conclusiones. Otros datos palinológicos refieren la existencia de polen de vid espontánea en el Pleistoceno medio en la localidad granadina de Padul (Florschutz et alii, 1971) así como durante el Paleolítico Superior de la Cueva de los Calaveres de Alicante (Fumanal y Dupré, 1983), y de la Cueva de les Mallaetes (Valencia) entre el 27000 y 29000 BP (Dupré, 1980).

Interesante resulta en este sentido la recopilación realizada por Walker (1984) para todo el Mediterráneo.

En segundo lugar, debemos referirnos a los táxones propios de medios ribereños. Resultado del diagrama podemos concluir la inexistencia de formaciones riparias densas, fenómeno atribuíble a una paralela degradación antrópica del bosque ripario, aunque no debe olvidarse la gran capacidad erosiva del río, por su encajamiento y el fuerte condicionamiento climático.

No obstante, táxones tales como las Ranunculaceae, Juncaceae, Rumex, Lycopodium, etc., ponen de manifiesto la presencia de una vegetación herbácea anexa a la ribera. La presencia constante a lo largo de todo el perfil de Polypodium puede ser indicativa de condiciones de mayor humedad ambiental y edáfica. De hecho, este elenco de especies de ámbitos higrófilos aparecen puntualmente en un mismo momento del diagrama polínico $(158 \mathrm{~cm})$, indicativo de ese microclima más húmedo.

La presencia de Alnus y Tilia, excepcionalmente puntual, debe ser causa de una contaminación o aporte exógeno en base a la dispersión del polen por el viento.

Asquerino (1987) cita la presencia durante el Atlántico de Jaen (5490 BP) de una serie de táxones arbóreos caducifolios tales como Tilia, Juglans, Populus y Ulmus. Tanto el tilo como el chopo, desaparecen con la llegada del Subboreal apareciendo por contra polen de haya (3990 BP). La ausencia en nuestros resultados de tales hechos nos llevan a considerar estas conclusiones.

Finalmente, debemos citar el numeroso y variado elenco de táxones de marcado carácter nitrófilo que aparecen en el diagrama. Su presencia puede ser debida bien a la propia acción degradativa del hombre o bien al carácter nitrófilo que éste determina. Carduaceae, Chenopodiaceae, Cichoriae, Plantago, Convolvulus, Malvaceae, etc. son reflejo de lo anteriormente expuesto.

Deben destacarse los altos porcentajes alcanzados por Carduaceae, que llegan hasta un $24 \%$ del P.N.A. y de Cichoriae que llegan al $70 \%$.

Respecto a especies propias del pastizal, cabe señalar la aparición de máximos alternantes que presentan Poaceae y Cyperaceae. Además, se observa una evolución coincidente en la presencia de Cyperaceae, Apiaceae, Cruciferae y Campanulaceae. La aparición de tales táxones junto a Carduaceae y Chenopodiaceae/Amaranthaceae, nos induce a pensar en una situación de pastizal abandonado, cuyo desarrollo tiene lugar tras la actividad humana. Cuando ésta se reanuda aumentan notablemente las Poaceae y disminuyen las Cyperaceae y, las especies nitrófilas ahora acompañantes, corresponden a $\mathrm{Ci}$ choriae y Centaureae.

En ambos casos, los porcentajes de Anthemideae son relativamente constantes.

En Córdoba, López García (1986) estudia el yacimiento de Ategua, de edad Subboreal-Subatlántico, que muestra un paisaje totalmente deforestado dominado por las Cichoriae y, con presencia importante de Cerealia y ruderales, estas últimas indicadoras de una fuerte actividad agrícola. Los aportes lejanos de A.P. corresponden a Pinus, Quercus, Buxus, Ulmus, Juglans, Juniperus y Populus. 
En nuestro caso, no se pone de manifiesto la presencia de polen de cereal.

Finalmente, deben citarse la existencia de dos táxones arbóreos: Pinus y Olea.

$\mathrm{La}$ aparición de polen de pino, en un porcentaje inferior al $10 \%$, poco significativo, y únicamente a partir del nivel $298 \mathrm{~cm}$, nos induce a pensar en una presencia alóctona de tal táxon en el diagrama.

La aparición de Olea se observa a partir de los $318 \mathrm{~cm}$, momento en que se supone comienza la sustitución del encinar por el olivar. Los porcentajes de Olea son ya constantes tras su primera presencia, coincidiento precisamente con los valores porcentuales más altos de Carduaceae y Fabaceae. No obstante, al aparecer un nivel estéril previo a esos $318 \mathrm{~cm}$., no nos permite precisar con rotundidad el momento de cultivo del olivo. Curiosamente, Olea desaparece a los $158 \mathrm{~cm}$, momentos antes de lo que lo hacen otros táxones $(138 \mathrm{~cm})$. Esta desaparición es fácilmente explicable en base a la eliminación del olivar para la construcción de viviendas, cuyos restos han quedado constatados arqueológicamente (Martín de la Cruz, 1978).

El reciente trabajo de Carrión (1991) en la cueva de Carihuela (Granada), cuyos depósitos pertenecen cronológicamente al Pleistoceno, hace referencia a la abundancia de Olea, que parece sugerir una extensión en las proximidades de la cavidad.

El yacimiento de Almizaraque, Almería (López García, 1986, 1988) muestra una secuencia polínica con una datación de $4090 \mathrm{BP}$, en la que Pinus domina los niveles inferiores. Los aportes de especies mediterráneas como Buxus, Vitis y Oleaceae no son nada despreciables. En los niveles superiores Pinus decrece y el P.N.A., en especial Cichoriae se hacen dominantes. $\mathrm{Ce}$ realia y otros táxones ruderales están presentes en la secuencia.

En resumen, el diagrama polínico estudiado es muy semejante al de Ategua (López García, 1986), aunque posiblemente más moderno, y por ello su cronología sea únicamente subatlántica.

A juzgar por nuestros resultados cabe destacarse el amplio desarrollo agrícola de los habitantes del yacimiento, lo que concuerda con la abundancia de restos arqueológicos romanos (Martín de la Cruz, 1978). Se puede pues afirmar que la romanización caló pronfundamente en los habitantes indígenas del Llanete de los
Moros o bien, son testimonio del asentamiento de colonos latinos en dichas tierras. Quizás esto último sea lo más acertado, por lo que podríamos pensar en un poblado ibérico pero con cierta cantidad de latinos instalados en él.

\section{BIBLIOGRAFÍA}

Asquerino, M.D. (1987): “Contribución de la Palinología a la reconstrucción del medio en la Prehistoria andaluza: La Cueva del Nacimiento (Pontones, Jaén)". Anales de la Asociación de Palinólogos de Lengua Española,3: 91-100.

CARrıón, J.S. (1991): "Desarrollo de vegetaciones mediterráneas durante el Pleistoceno superior en el sureste ibérico. Nuevos datos polínicos". Anales de Biología, 17: 109-131.

Couteaux, M. (1977): “A propos de l'interpretation des analyses polliniques de sédiments minéraux, principalment archéologiques". In H. Laville, \& J. RenaultMiskovsky (eds.): Approche écologique de l'homme fossile. Suppl. de l'A.F.E.Q., Paris: 259-276.

Diaz Fernández, P.M. (1992): "Historia de la Vegetación de Sierra Madrona y su entorno". Memoria de Licenciatura (inédit.). Universidad Complutense. Madrid.

DUPRE, M. (1979): “Breve manual de análisis polínico". Instituto Juan Sebastián Elcano. C.S.I.C. Departamento de Geografía. Universidad de Valencia.

- (1980): "Análisis polínico de sedimentos arqueológicos de la Cueva de les Malladetes (Barx, Valencia)". Cuadernos de Geografía, 26: 1-22.

Florschutz, F.; Menéndez Amor, J. and Wijmstra, T.A. (1971): "Palynology of a thick Quaternary succesion in southern Spain". Palaeogeography, Palaeoclimatology and Palaeoecology, 10: 233-264.

Fumanal, M.P. et DuPre, M. (1983): "Schéma paléoclimatique et chrono-statigraphique d'une séquence du paléolitique supérieur de la région de Valence, Espagne". Bulletin de l'Association Française pour l'Etude du Quaternaire, 1: 39-6.

Goeury, C.L. et Beaulieu, J.L. (1979): “A própos de la concentration du pollen a l'aide de la liqueur de Thoulet dans les sédiments minéraux". Pollen et Spores, 21 (1-2): 239-251.

Gómez Mercado, F. \& Valle Tendero, F. (1988): “Mapa de Vegetación de la Sierra de Baza". Universidad de Granada.

López García, P. (1978): "Resultados polínicos del Holoceno en la Península Ibérica". Trabajos de Prehistoria, 35: 9-44.

- (1984): "Aplicaciones de la Palinología a la Prehistoria: métodos utilizados y resultados”. Actas Primeras Jornadas de Metodología de Investigación Prehistórica (Soria, 1981). Ministerio de Cultura. Madrid: 309-317. 
- (1986): "Estudio Palinológico del Holoceno Español a través del análisis de yacimientos arqueológicos". Trabajos de Prehistoria. 43: 143-158.

- (1988): "Estudio polínico de seis yacimientos del sureste español". Trabajos de Prehistoria, 45: 335-345.

MaRTIN DE la CRUz, J.C. (1978-9): "Montoro. Un nuevo yacimiento arqueológico en el Guadalquivir". Cuadernos de Prehistoria y Arqueologia, 5-6: 105-141.

- (1984-5): "Problemas en torno a la definición del Bronce tardío en la Baja Andalucía". Cuadernos de Prehistoria y Arqueología, 11-12: 205-215.

- (1987): El Llanete de los Moros. Montoro, Córdoba. Excavaciones Arqueológicas en España. 151.

MAPA (1977): "Mapa de cultivos y aprovechamientos de la provincia de Córdoba". Inventario Forestal Nacional del Ministerio de Agricultura. Madrid.

Menéndez Amor, J. y Florschutz, F. (1964): "Resultados del análisis paleobotánico de una capa de turba en las cercanías de Huelva (Andalucía)". Estudios Geológi$\cos , 20$ : 183-186.

MOORE, P.D. \& WEBB, J.A. (1978): “An illustrated guide to Pollen Analysis". Hodder \& Stroughton. London.

Moore, P.D.; Webr, J.A. and Collinson, M.E. (1991): "Pollen analysis". Blackwell Scientific Publications. London.
Peinado lorca, M. y Rivas Martinez. S. (1987): "La Ve. getación de España". Universidad de Alcalá de Henares. Madrid.

Rivas-Martínez, S. (1987): “Memoria del MAPA de Series de Vegetación de España". Ministerio de Agricultura, Pesca y Alimentación. ICONA. Madrid.

Sanchez MatA, D. y de la Fuente, V. (1986): “'Las riberas de agua dulce". Unidades Temáticas Ambientales. Dirección General de Medio Ambiente. MOPU. Madrid.

Stevenson, A.C. (1985): "Studies in the vegetational history of S.W. Spain. II. Palynological investigations at Laguna de las Madres, S.W. Spain". Journal of Biogeography, 12: 293-314.

Stevenson, A.C. \& Moore, P. (1988): "Studies in the vegetational history of S.W. Spain. IV. Palynological investigations of a valley mire at El Acebron, Huelva". Journal of Biogeography, 15: 339-361.

VAZQUEZ, R. (1992): "Evolución del paisaje vegetal durante el Cuaternario reciente en la zona central y oriental de la Sierra de Guadarrama a partir del análisis polínico". Tesis doctoral (inéd.). Universidad de Alcalá de Henares. Facultad de Ciencias. Madrid.

WALKER, M.J. (1984): "Nuevos datos acerca de la explotación de la vid en el eneolítico español". Cuadernos de Prehistoria y Arqueología, 11-12 (1): 163-182. 